\title{
Computed tomography guided fine needle aspiration cytology of thoracic lesions: A retrospective analysis of 114 cases
}

\author{
Jacob Baby*, Paul George** \\ *Associate Professor, Department of Pulmonary Medicine \\ ** Associate Professor, Department of Pathology Cochin Medical College Kochi, Ernakulam, Kerala, India
}

\begin{abstract}
Objectives: The purpose of the current study was to retrospectively analyze the age, sex, topographic distribution, the size, cytopathological diagnosis, the diagnostic accuracy and complications of thoracic mass lesions using CT-guided FNAC. The results will be compared with other published studies

Materials and Methods: Retrospective hospital based descriptive study in Tertiary care setting in an Indian hospital. The clinical, radiological and cytological data of 114 patients were studied who underwent CT guided FNAC from Nov 2008 to Aug 2013.

Results:- Of the 114 cases there were 96 parenchymal lesion, hilar 13, mediastinal 4 and one pleural. Definitive cytological diagnosis was obtained in 100 cases. Diagnostic accuracy in our study was $87.7 \% .43$ cases were malignant of which Adenocarcinoma was the most common type. 22 cases were diagnosed as lesions that were consistent with Tuberculosis. 3 cases developed pneumothorax (3.14\%) of which one required treatment and 2 cases(2.28\%) had hemoptysis.

Conclusion CT guided fine needle aspiration cytology (FNAC) is a simple and safe procedure with high diagnostic accuracy for the diagnosis of Lung cancer. Has great potential in confirming smear negative pulmonary Tuberculosis.
\end{abstract}

Key words: CT guided, percutaneous, thoracic mass, cytology

\section{Introduction}

Percutaneous, transthoracic fine needle aspiration cytology (FNAC) is a well established diagnostic method used in the cytological evaluation of thoracic mass lesions for the last three decades. Haaga and Alfidi reported computed tomography (CT)-guided biopsy in 1976. Reports since that time have shown that Transthoracic Needle Aspiration Cytology or Biopsy procedures are both effective and accurate.. This procedure provides a safe, rapid, and accurate diagnosis in patients having thoracic mass lesions. ${ }^{(1,2,3)}$ In cases of malignancy of the lungs, cytopathological examination of material obtained by CT-guided FNAC offers a quick and specific diagnosis. This will help clinicians implement appropriate anticancer measures like chemotherapy and radiotherapy. It has also been demonstrated in literature that CT-guided FNAC is an accurate and sensitive way of diagnosing malignancy of the lungs. ${ }^{(3,4,5)}$ On the other hand, post procedure complications are fewer except for pneumothorax, pulmonary hemorrhage, and hemoptysis in a small percentage of cases.

Objective of the study:-The purpose of the current study was to retrospectively analyze the age, sex, and topographic distribution, the size, cytopathological diagnosis, the diagnostic accuracy and complications of thoracic mass lesions using CT-guided FNAC. The results will be compared with other published studies.

\section{Materials and methods}

Study setting: - Tertiary care setting- Department of Pulmonary Medicine of a teaching hospital in India. Study period- Nov 2008 to Aug 2013

Significance of the study: - Majority of the studies published is from the radiology department. Very few chest departments are actively doing CT guided FNAC. The Guidelines published by British Thoracic Society suggest the involvement of the respiratory physician. ${ }^{7}$ All the FNAC done in this study was by the chest physician.

Inclusion criteria

After institutional approval the radiological and cytological information of 114 patients were obtained from the hospital data who underwent CT guided FNAC from Nov 2008 to Aug 2013

The criteria for patient selection were as follows:

1. Patient was cooperative and was able to hold breath for a short while.

2. Patient had no bleeding tendency or coagulopathy.

Exclusion criteria ${ }^{8}$

1. Severe chronic obstructive pulmonary disease (FEV1<30\% predicted/Respiratory failure)

2. A bleeding disorder (including drug-induced bleeding tendency).

3. A contralateral pneumonectomy . 
4. Pulmonary arterial hypertension .

Investigations done before the procedure

1. BT, CT, platelet count.

2. Oxygen saturation.

FNAC was done after informed consent- benefits \& reminding chance of hemoptyis and pneumothorax, which are treatable. The procedure is as follows.

First an axial scan of the area of interest will be done to locate the lesion.

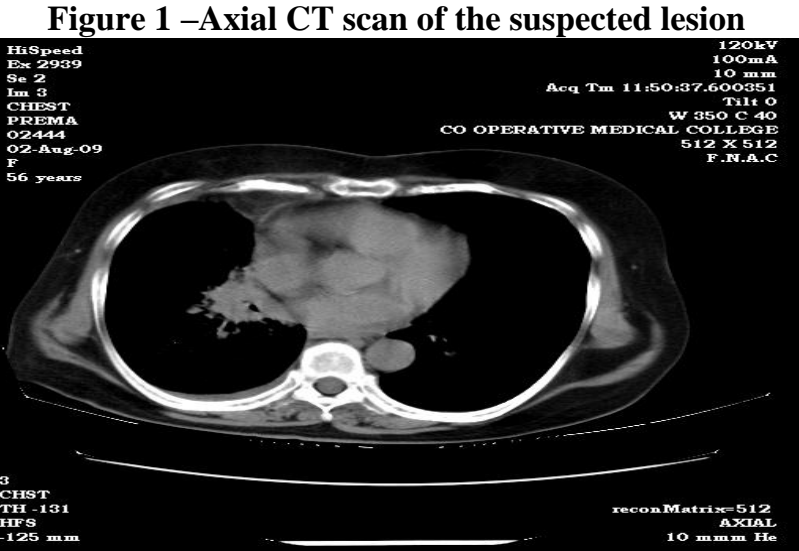

Best approach (Supine or Prone) is judged .The skin puncture site is marked with a radio opaque marker. CT scan done with the marker.

Figure 2 - CT scan with radio opaque marker to measure the distance and guide needle entry

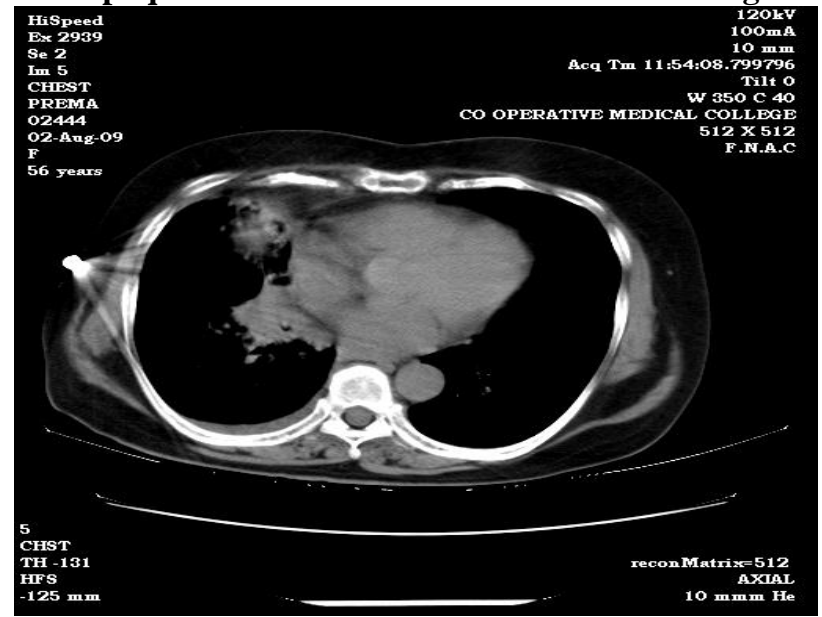

The distance is measured from the skin surface to the lesion. After cleaning the skin surface with povidone iodine and draping, local anesthetic (2\% Xylocaine) was infiltrated at the site of puncture. The 20-gauge spinal needle is then inserted during suspended respiration, directing the tip of needle towards the lesion. With the tip of the needle located in the lesion, a repeat slice of the area of interest was taken to check the exact position of its tip.

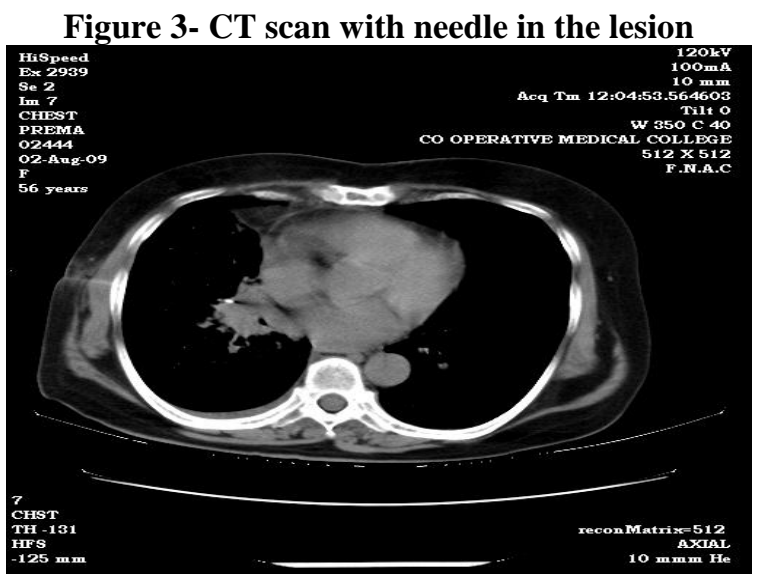


The stylet is then withdrawn $2-3 \mathrm{~cm}$ and the needle advanced into the mass with a rotating motion during suspended respiration, so that its tip lies within the target lesion. 20-ml syringe is attached to the needle's hub and the plunger pulled back, and during continued hard suction, the needle is jiggled to free material from the lesion to the needle's lumen. Five to ten smears are prepared immediately from the sample in the CT scan room. Usually two specimens are kept as dried specimens and three to four slides will be fixed immediately in $95 \%$ alcohol. Air-dried smears were stained with May-Grünwald-Giemsa stain whereas alcohol-fixed smears were stained with Papanicolaou and hematoxylin and eosin stains for rapid cytopathological evaluation of the lesions. A follow-up CT scan was done in every patient immediately after the procedure to rule out pneumothorax.

\section{Results}

Data was compiled and statistical analysis was done by using Statistical Package for the Social Sciences (SPSS) version 10.0 for windows

Of the 114 cases 15 were females $(17.1 \%)$ and $99(86.8 \%)$ males.

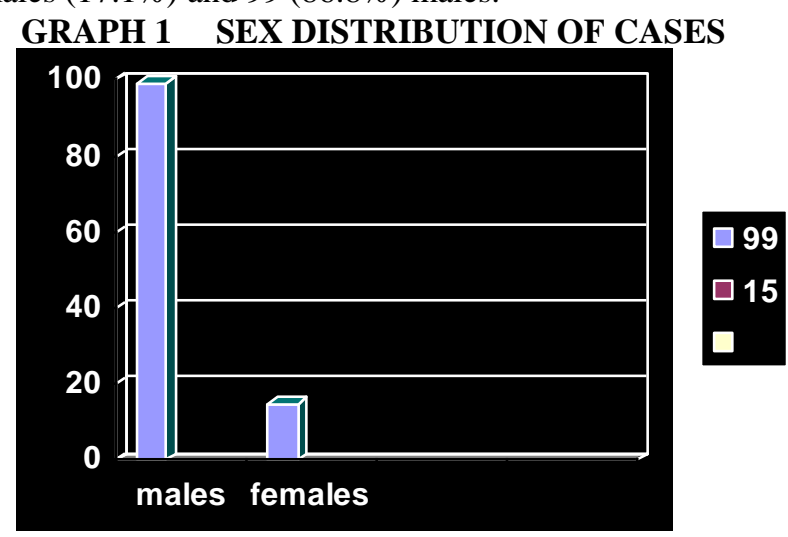

\section{GRAPH 2 CHART SHOWING THE AGE DISTRIBUTION OF THE CASES}

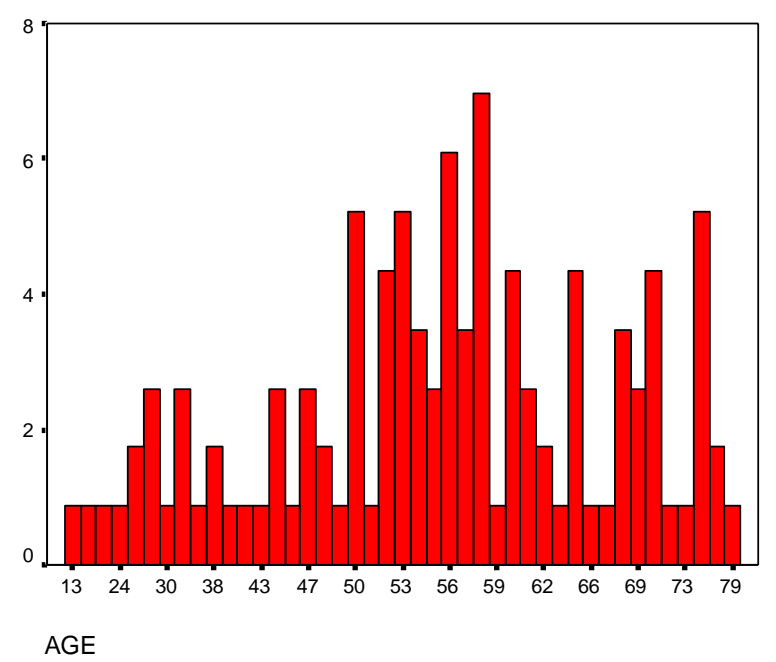

Age of the patients ranged from 13 to 79 with a mean age of 46. Maximum age group was in the 50- 59 group. 37 cases were on the Left side and 67 on Right side. Regarding the topographic distribution it was as follows. Hilar 13, Mediastinal- 4, Parenchymal-96 Pleural- 1.

Out of 114 cases of thoracic mass lesions, definitive cytological diagnosis was obtained in 100 cases and the rest of cases were inconclusive and descriptive report was given to the patients. Accuracy in our study for diagnosis $-87.7 \% \quad(100 / 114)$ 
Table 1: Cytopathological spectrum of the CT Guided FNAC lesions

\begin{tabular}{|c|c|c|}
\hline Adenocarcinoma & 19 & 16.7 \\
\hline Squamous cell $\mathrm{Ca}$ & 14 & 12.3 \\
\hline Small cell $\mathrm{Ca}$ & 5 & 4.4 \\
\hline Small cell anaplastic $\mathrm{Ca}$ & 1 & 0.9 \\
\hline Bronchiolalveolar Ca & 1 & 0.9 \\
\hline Large cell $\mathrm{Ca}$ & 1 & 0.9 \\
\hline Poorly differentiated NonsmallCell & 1 & 0.9 \\
\hline Non Hodgkins Lymphoma & 1 & 0.9 \\
\hline Hodgkins Lymphoma & 1 & 0.9 \\
\hline Squamous metaplasia & 1 & 0.9 \\
\hline Mesothelioma & 1 & 0.9 \\
\hline Atypical cells & 9 & 7.9 \\
\hline Chronic inflammatory cells & 8 & 7.0 \\
\hline Acute on chronic inflammatory & 2 & 1.8 \\
\hline Pneumonia & 1 & 0.9 \\
\hline Rheumatoid & 1 & 0.9 \\
\hline Sarcoidosis & 1 & 0.9 \\
\hline Kochs & 22 & 19.3 \\
\hline Aspergillus & 2 & 1.8 \\
\hline Subacute inflammation & 1 & 0.9 \\
\hline Blood & 3 & 2.6 \\
\hline Epitheliod cells & 1 & 0.9 \\
\hline Inflammatory cells & 17 & 14.9 \\
\hline
\end{tabular}

Among 100 cytologically diagnosed cases, 43 cases were malignant (including both lung and mediastinal tumors).

Total $45 / 100(45 \%)$

Table 2 -Histopathological subtypes of Malignant lesions.

Of the malignant cases Adenocarcinoma tops the list, followed by Squamous and Small cell carcinoma.

\begin{tabular}{|l|ll|}
\hline Adenocarcinoma & 19 & $(42 \%)$ \\
\hline Squamous cell carcinoma & 14 & $(31 \%)$ \\
\hline Small cell carcinoma & 6 & $(13 \%)$ \\
\hline Lymphoma & 1 & $(2 \%)$ \\
\hline Poorly differentiated non small cell & 1 & $(2 \%)$ \\
\hline Large cell & 1 & $(2 \%)$ \\
\hline Bronchioloalveolar carcinoma & 1 & $(2 \%)$ \\
\hline Nonhodgkins lymphoma & 1 & $(2 \%)$ \\
\hline Mesothelioma & 1 & $(2 \%)$ \\
\hline
\end{tabular}

\section{Diagnosis of Tuberculosis in Smear negative cases}

22

were

diagnosed as

that were

consistent

tuberculosis.

\begin{tabular}{|l|l|}
\hline Type of lesion & No. \\
\hline Granuloma with langerhans giant cells & 22 \\
\hline Epitheloid cells & 1 \\
\hline Chronic inflammatory cells & 8 \\
\hline
\end{tabular}

cases

lesions

with

Granuloma with Langerhans giant cells or chronic inflammation suggestive of tuberculosis 


\section{Complications}

Complications observed were pain at the puncture site, pneumothorax and hemoptysis. Pneumothorax was observed in three cases. All the pneumothorax cases were diagnosed by the repeat CT scan immediately after the procedure. One case required Intercostal Tube drainage. All the three cases the lesion was more than 20 $\mathrm{mm}$ from the pleura. Hemoptysis was observed in 2 cases.

\section{Discussion}

Transthoracic fine needle aspiration cytology (FNAC) of thoracic lesion using CTguidance is a relatively safe and accurate means of diagnosing benign and malignant lesions of the chest with negligible mortality. The reported accuracy in the literature ranged from $64 \%$ to $97 \% .^{9}$ Diagnostic accuracy of our study was $87.7 \%$.The accuracy was comparable to the recent studies published.

TABLE 4 Summary of results of reported series of CT-guided FNAC

\begin{tabular}{|l|l|l|l|l|l|}
\hline References & No. of cases & Diagnostic accuracy & Pneumothorax & Hemorrhage & Hemoptysis \\
\hline Stanley et al 1987 & 458 & $96.6 \%$ & $133(29 \%)$ & - & $5(1.1 \%)$ \\
\hline Vansonnenberg et al 1988 & 150 & $82.7 \%$ & $64(42.7 \%)$ & 2 & $5(3.3 \%)$ \\
\hline Haramati et al 1995 & 32 & $81 \%$ & $3(9.4 \%)$ & - & - \\
\hline Santambrogio et al 1997 & 110 & $81 \%$ & $23(20.9 \%)$ & - & - \\
\hline Gouliamos et al 2000 & 64 & $98.4 \%$ & $2(3.1 \%)$ & 1 & - \\
\hline Mohammad et al 2001 & 184 & $97 \%$ & $2(1.1 \%)$ & & $10(5.4 \%)$ \\
\hline Gupta et al2002 & 37 & $91 \%$ & $1(2.7 \%)$ & 1 & \\
\hline $\begin{array}{l}\text { J P Singh et al 2004 } \\
\text { Su }\end{array}$ & & $85.3 \%$ & $4(11.8 \%)$ & 4 & $1(2.9 \%)$ \\
\hline $\begin{array}{l}\text { Present series } \\
2009\end{array}$ & 114 & $87.7 \%$ & & & $2(2.28 \%)$ \\
\hline
\end{tabular}

Males formed the majority 99(86.8\%).This may be because of predominance of malignancies in the male sex. This is similar to one published recently ${ }^{10}$. Youngest patient was a male with 13 years and oldest also a male of 79 years . The study showed that the maximum number of cases was seen in the age group of 50-59 years. The increased number of cases in the age group of 50-59years may be due to increased incidence of malignancies in that group and also because FNAC was mainly used for the diagnosis of neoplasm, which comprises $43 \%$ of the total cases. The average age in our study was 46 years. Maxcy Roseau's last study indicated that an exponential increase in the incidence rates with age is observed for most adult malignancies. This is true as increased number of malignant cases is seen in the elderly population.

Regarding the side of the lesions majority were on the right side 67 cases (59\%) .This is comparable with other published studies ${ }^{8}$ When we analyzed the distribution it showed that majority were parenchyma 94$(82 \%)$ followed by Hilar -13 .This is because the cases selected were predominantly pulmonary nodules which were accessible by needle.

Cytological study showed a malignant pathology in $43 \%$ of cases and a benign Pathology in 57\% cases. The prevalence of malignancy in our study is significantly less than the $81.8 \%$ found in similar study done by Singh et al. and the reported $62 \%$ in a study by Basnet et al ${ }^{12}$ Of the malignant cases Adenocarcinoma -19 cases $(42 \%)$ were more than Squamous cell 14 cases(31\%).The reported increase in Adenocarcinoma (42\%)differs from the reported series by Singh et al $22 \%$ and higher than reported by Basnet et al $(12 \%)^{\mathbf{1 2}}$. This difference may be due to the rising trend of Adenocarcinoma in our state.

An interesting finding in our study was the prevalence of benign cases mainly contributed by Tuberculosis cases. We had 22 cases of Tuberculosis diagnosed cytologically by the presence of granulomas with Langerhans giant cells. Of these , 4 cases had evidence of tubercle bacilli on AFB staining of the dried smears. One case interestingly showed evidence of adenocarcinoma with Granuloma and Tubercle bacilli. The rest of cases responded very well to Anti Tubercular Treatment. Thus CT guided FNAC can be a valuable tool to diagnose Smear negative pulmonary Tuberculosis.

Regarding the complications of CT guided FNAC the most common reported is pain at the site of puncture which was observed in majority. The other complications are pneumothorax and hemoptysis. 
Pneumothorax is, by far, the most frequent complication of the procedure: Reported rates range widely, from $5 \%$ to $61 \%{ }^{13,14}$. Most of these data pertain to fluoroscopic guided TNAB. The reported rate of pneumothorax with CT-guided biopsy may be slightly higher because CT is more sensitive for detection of pneumothorax. Our series reported 3 cases of pneumothorax (3.14\%). Pneumothorax was significantly lower than the $42.7 \%, 29 \%$, $20.9 \%$ and $11.8 \%$ seen in the studies conducted by Vansonnenberg et al ${ }^{15}$, Stanley et $\mathrm{al}^{\mathbf{1 6}}$, Santambrogio et $\mathrm{al}^{18}$ and Singh et al ${ }^{8}$ respectively. It was comparable similar to the $3.1 \%, 2.7 \%$ and $1.1 \%$ of Gouliamos et al ${ }^{19}$, Gupta et al ${ }^{20}$ and Mohammad et al ${ }^{9}$. One case which required Intercostal Tube drainage had underlying COPD. Further analysis into these cases showed that they had more than one pass into the pleura .In two cases the distance between the lesion and pleura were more than $2 \mathrm{~cm}$. This is in accordance with other studies and published guidelines by BTS. ${ }^{7}$. The incidence of pneumothorax can be minimized by reducing the number of passed into the pleura and also avoiding the fissures ${ }^{7}$

Verification of cytological diagnosis was made by histopathological examination wherever possible and therapeutic response to relevant therapy in the remaining cases.

Limitation of our series is that the follow up of the malignant cases could not be completed as they were lost to follow up after being referred to the oncology division at another hospital.

\section{Conclusion}

CT guided fine needle aspiration cytology (FNAC) is a simple and safe procedure with high diagnostic accuracy for the diagnosis and cell typing of Lung cancer .It also has great potential in confirming smear negative pulmonary Tuberculosis cases. Though complications are rare, pneumothorax, hemoptysis and chest pain are occasionally encountered, but rarely require active management.

\section{References}

[1]. Stewart CJ, Stewart IS. Immediate assessment of fine needle aspiration cytology of lung. J Clin Pathol 1996;49:839-43.

[2]. Salazar AM, Westcott JL. The role of transthoracic needle biopsy for the diagnosis and staging of lung cancer. Clin Chest Med 1993;14:99-110.

[3]. Sanders C. Transthoracic needle aspiration. . Clin Chest Med $1992 ; 13: 1-16$.

[4]. Mullan CP, Kelly BE, Ellis PK, Hughes S, Anderson N, McCluggage WG. CT-guided fine-needle aspiration of lung nodules: Effect on outcome of using coaxial technique and immediate cytological evaluation. Ulster Med J 2004;73:32-6.

[5]. Cox JE, Chiles C, McManus CM, Aquino SL, Choplin RH. Transthoracic needle aspiration biopsy: Variables that affect risk of pneumothorax.Radiology 1999; 212:165-8.

[6]. Ko JP, Shepard JO, Drucker EA, Aquino SL, Sharma A, Sabloff B, et al. Factors influencing pneumothorax rate at lung biopsy: Are dwell time and angle of pleural puncture contributing factors? Radiology 2001;218:491-6.

[7]. A manhire,M Charig, C Clelland, R Miller, H Moss,K Pointon,C.Richardson and E Sawicka. Guidelines for radiologically guided lung biopsy .Thorax 2003; 58: 920-936

[8]. Sing JP. Garg L, Setia V. Compared tomography (CT) guided transthoracic needle aspiration cytology in difficult thoracic mass not approachable by USG. Indian J Radiol Imaging 2004;14:395-400.

[9]. Mohammad GM. CT guided fine needle aspiration cytology in the diagnosis of thoracic lesions. JIMA 2001; 99(10): 1-5

[10]. Pandey DK, Ahmad Z, Masood I, Singh SK, Jairajpuri Z. Role of fine-needle aspiration cytology in evaluating mediastinal masses. Lung India 2009; 26: 114-6

[11]. Maxcy Rosenau-last: Public health and preventive medicine. Appleton and Lange; 1993.

[12]. Computed Tomography Guided Percutaneous Transthoracic Fine Needle Aspiration Cytology in Chest Masses. Basnet SB, Thapa GB, Shahi R, Shrestha M, Panth J Nepal Med Assoc 2008;47(171):123-7

[13]. Herman PG, Hessel SJ. The diagnostic accuracy and complications of closed lung biopsies. Radiology 1977; 125:11-14.

[14]. Poe RH, Kallay MC, Wicks CM, Odorof CL. Predicting risk of pneumothorax in needle biopsy of the lung. Chest1984; 85:232-235

[15]. VanSonnenberg E, Casola G, Ho M, et al. Difficult Thoracic Lesions: CT-guided biopsy Experience in 150 cases. Radiology 1988; 167: 457-461

[16]. Stanley JH, Fish GD, Andriole JG, et al. Lung lesions:cytological diagnosis by fine needle biopsy. Radiology 1987; 162:389-91.

[17]. Haramati LB. CT-guided automated needle biopsy of the chest. AJR 1995; 165: 53-55.

[18]. Santambrogio L, Nosotti M, Bellaviti N, Pavoni G, Radice F, Caputo V. CT guided fine needle aspiration cytology of solitary pulmonary nodules: a prospective, randomized study of immediate cytologic evaluation. Chest 1997; 112:423-5.

[19]. 19. Gouliamos AD, Giannopoulos DH, Panagi GM, Fletoridis NK, Deligeorgi-Politi HA, Vlahos LJ. Computed tomography-guided fine needle aspiration of peripheral lung opacities: An initial diagnostic procedure .Actacytologica 2000; 44 (3): $344-348$.

[20]. Gupta S, Michael JW, Frank AM, Kamran A, Marshall EH. CT guided percutaneous needle biopsy of intrathoracic lesions by using the transternal approach: experience in 37 patients. Radiology 2002; 222: 57-62 